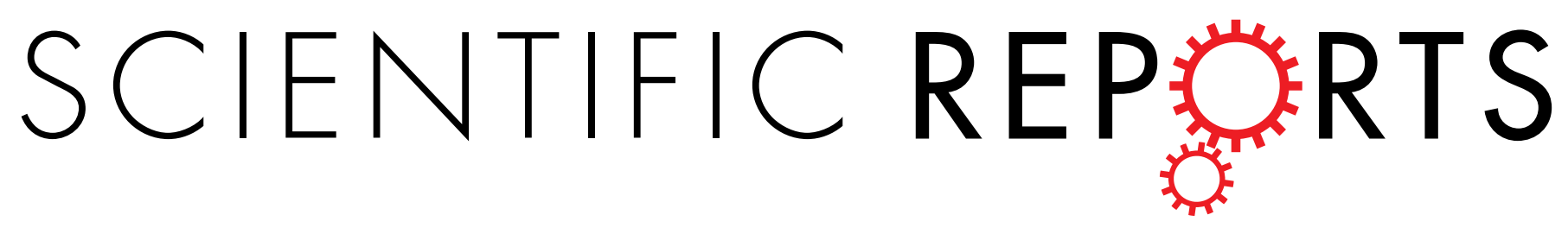

\title{
Corrigendum: Structural and functional characterization of a novel scFv anti-HSP6o of Strongyloides sp.
}

Marcelo Arantes Levenhagen, Fabiana de Almeida Araújo Santos, Patrícia Tiemi Fujimura, Ana Paula Carneiro, Julia Maria Costa-Cruz \& Luiz Ricardo Goulart

Scientific Reports 5:10447; doi: 10.1038/srep10447; published online 21 May 2015; updated on 05 August 2015 The original version of this Article contained a typographical error in the spelling of the author Ana Paula Carneiro, which was incorrectly given as Ana Paula Caneiro. This has been corrected in the PDF and HTML versions of the Article. 